\title{
LA PRUEBA DE COHEN: CULMINACIÓN DE LA CRISIS EN LA AXIOMÁTICA
}

\section{Introducción}

A finales de 1963 y principios de 1964 se ha producido un acontecimiento de importancia fundamental en la historia de la matemática y la lógica, con la publicación del trabajo de Paul J. Cohen sobre la independencia de la hipótesis del continuo respecto a los otros axiomas de la teoría de los conjuntos. ${ }^{1}$ En dicho trabajo, el joven matemático de la Universidad de Stanford ha realizado la hazaña extraordinaria de dividir la teoría de los conjuntos, precisamente de la misma manera como Lobachevski consiguió establecer en 1826 la división de la geometría en euclidiana y no-euclidiana. Además, hace tiempo se puso al descubierto que la teoría de los conjuntos constituye la estructura primordial en la cual se apoyan los otros desarrollos de la matemática, hasta el punto de que "todas las teorias matemáticas pueden ser consideradas como extensiones de la teoría general de los conjuntos".2 Por lo tanto, el trabajo de Cohen significa la iniciación de una revolución en la matemática entera, cuyos resultados llegarán a tener alcances incalculables. Al mismo tiempo, debido a que la teoría de los conjuntos representa la estructura matemática fundamental de la lógica, es claro que el descubrimiento de Cohen producirá también una transformación profunda y de graves consecuencias en el seno de la lógica. En particular, la prueba encontrada por Cohen viene a ser la culminación de la crisis suscitada por los trabajos de Goedel en relación con la formalización axiomática de la ciencia y, a la vez, con ella se orienta el desenlace de esa crisis en un sentido definido.

En este ensayo trataremos de establecer las consecuencias inmediatas y las perspectivas que ofrece la prueba de Cohen. Para ello, empezaremos por hacer una breve relación de los antecedentes del problema. Luego examinaremos el contexto en el cual se plantea. En seguida nos referiremos a las pruebas de Goedel, de las cuales es un complemento directo la demostración de Cohen, a la vez que representa la solución de la principal cuestión que aquellas dejaron abierta. Finalmente, señalaremos los resultados que son inherrentes al descubrimiento mismo, analizaremos las consecuen-

1 "The Independence of the Continuum Hypothesis", Proceedings of the National Academy of Sciences, Washington, 50, 1963, págs. 1143-1148; 5I, 1964, págs. $105^{11} 10$.

2 Nicolas Bourbaki, Éléments de Mathématique, Théorie des Ensembles, París, Hermann, 1939 . 
cias más destacadas que trae aparejadas y mostraremos las amplias perspectivas que presenta.

\section{El método axiomático}

La conocida disposición utilizada por Euclides en sus Elementos para exponer demostrativamente la geometría, constituye la primera exposición axiomática de una disciplina científica. En esa exposición se enuncian 23 definiciones, 5 postulados y 9 nociones comunes, cuya validez se admite como evidente, para probar después los teoremas geométricos por medio de inferencias correctas basadas en ellos. Por consiguiente, en la exposición euclidiana, todos los teoremas son implicados por o deducidos del grupo de axiomas constituido por las nociones comunes, los postulados y las definiciones. Con seguridad, el método empleado por Euclides fue el procedimiento concreto consistente en tomar el cuerpo de conocimientos establecidos én su época y formularlos sistemáticamente, para lo cual idealizó los conceptos implicados y seleccionó entre las relaciones conocidas un pequeño número de principios elementales, de los cuales se pudieran derivar todos los demás por deducción. Ese mismo procedimiento fue utilizado por Newton para sistematizar la mecánica. Dicho método axiomático concreto es un poderoso instrumento racional que es aplicable en el momento en que una ciencia está tomando forma, o sea, cuando ya son discernibles las leyes generales que gobiernan los procesos que estudia. En tal caso, la tarea consiste simplemente en hacer una colección completa de los conceptos básicos o categorías de una ciencia y de sus relaciones fundamentales, de las cuales se puedan derivar por definición los otros conceptos y, por deducción, los teoremas. De esa manera, el método axiomático permite comprender mejor la ciencia en su conjunto, al separar claramente las deducciones lógicas rigurosas, de las consecuencias concretas que de ellas se desprenden y las cuales se apoyan en una determinada interpretación concreta de ese esquema axiomático.

Por otra parte, durante varios siglos se hicieron esfuerzos considerables para encontrar la demostración de los axiomas euclidianos y, en particular, del quinto postulado relativo a las paralelas. Como es sabido, tales esfuerzos culminaron en un resultado justamente opuesto al buscado, como fue la prueba encontrada por Lobachevski de la imposibilidad de demostrar dicho postulado o, lo que es lo mismo, de su independencia respecto a los otros axiomas. ${ }^{3}$ Debido a su independencia, el postulado de las paralelas pudo ser

3 Nicolai I. Lobachevski, Pangéométrie ou précis de Géométric fondée sur une théorie générale et rigoureuse des parallèles, incluido en Collection complète des oeuvres géométriques de N. I. Lobatcheffsky, Kazán, vol. II, págs. 615-680. (Pangeometria, Suplementos del Seminario de Problemas Científicos y Filosóficos, México, U.N.A.M., Núm. 10, primera serie, traducción de Eli de Gortari, 1956.) 
sustituido por su negación contradictoria, tanto en el sentido de postular que por un punto exterior a una recta pasan dos o más paralelas, en vez de una sola, como en el de postular que no pasa ninguna paralela. Entonces, introduciéndo este nuevo postulado, se construyeron las geometrías no-euclidianas y se puso en claro que la demostración formal tiene que detenerse en ciertas proposiciones admitidas sin prueba, para no proseguir en un procedimiento infinito. Con base en lo anterior, se fortalecieron los propósitos de formalización y se realizó un análisis lógico penetrante y sumamente riguroso de los fundamentos de la geometría y la aritmética. Así fue como Peano estableció un sistema formado por 5 axiomas para inferir, a partir de ellos, todos los teoremas de la aritmética. ${ }^{4}$ Igualmente, Hilbert estableció un sistema constituido por 20 axiomas, para derivar de él todos los teoremas de la geometría elemental. ${ }^{5}$ Por su parte, Zermelo formuló la axiomatización de la teoría de los conjuntos. ${ }^{6} \mathrm{Y}$ finalmente, en esa etapa inicial de la axiomática, Whitehead y Russell establecieron la formalización de los fundamentos de la matemática. ${ }^{7} \mathrm{Al}$ propio tiempo, se encontró que las relaciones lógicas expresadas por los axiomas también se cumplen para otros muchos conceptos diferentes, o sea, que la validez de las demostraciones se funda en la estructura de las proposiciones axiomáticas, más que en algún contenido particular de los conceptos relacionados por ellas. Por lo tanto, se vino a considerar que los términos que figuran en los axiomas son "conceptos vacios", los cuales sólo adquieren el significado que les imparten las relaciones enunciadas en los axiomas primero y, luego, en las definiciones y los teoremas.

Con los resultados anteriores se desarrolló ampliamente la axiomatización de varias disciplinas matemáticas, formalizándolas en sistemas compuestos por cuatro tipos de proposiciones: axiomas, definiciones, reglas de operación y teoremas. Los axiomas son proposiciones elementales en las cuales se postulan relaciones primitivas entre conceptos no-definidos. $O$ sea, que los axiomas son definiciones implícitas en las que simplemente se declaran los conceptos no-definidos, mediante su inclusión en la relación primitiva que enuncian. Las definiciones introducen nuevos conceptos, que se definen en función de los conceptos no-definidos que figuran en los axiomas. Es decir, que las definiciones declaran explícitamente los nuevos conceptos que introducen, refiriéndolos a los conceptos no-definidos y sus relaciones primitivas. Por su parte, las, reglas de operación permiten construir nuevas proposiciones a partir de los axiomas y definiciones; para lo cual se utilizan los

4 Giuseppe Peano, Arithmetices Principia nova methodo exposita, Turin, 1889.

5 David Hilbert, Grundlagen der Geometrie, Leipzig, 1899. (Fundamentos de la Geometria, Madrid, Consejo Superior de Investigaciones Cientificas, traducción de F. Cebrián, 1953.)

6 Ernst Zermelo, "Untersuchungen ueber die Grundlagen der Mengenlehre", Mathematische Annalen, 65, 1908, págs. 261-281.

7 Alfred North Whitehead y Bertrand Russell, Principia Mathematica, Cambridge, Inglaterra, 1910-1913, 3 vols. 
preceptos canónicos de.la identidad, la no-contradicción y la exclusión de tercero, junto con las reglas de inferencia de la deducción formal. Por último, los teoremas son las proposiciones que se obtienen directamente a partir de los axiomas y definiciones o, indirectamente, con apoyo en otros teoremas previamente demostrados, aplicando siempre las reglas de operación aceptadas.

De esta manera quedó constituido el método axiomático formal como instrumento para construir la estructura abstracta de las disciplinas científicas, empezando con los conceptos y relaciones primitivas de los axiomas, y desarrollando entonces sus consecuencias formales sin hacer referencia a ningún significado concreto ni específico. Lo que es más, se llegó a considerar que los axiomas son establecidos arbitrariamente $a$ priori, sin tener que recurrir para nada a las nociones intuitivas, la observación, la experimentación y, en general, la realidad objetiva. Entonces, una vez establecido el grupo de axiomas correspondiente a una disciplina, de ellos se deben poder derivar deductivamente todas las otras propiedades que la integran, expresándolas como teoremas. En todo caso, la demostración formal de cada teorema tiene que establecerse por medio de una secuencia finita de inferencias, cada una de las cuales tiene como premisas a los axiomas, o bien, a otros teoremas ya demostrados. En esas condiciones, el método axiomático suministra explicaciones lógicas sobre una disciplina, en tanto retrocede a sus relaciones primitivas y, luego, asciende de nuevo a las proposiciones derivadas, a través de una rigurosa consecuencia. De este modo se han descubierto graves incorrecciones lógicas que habían pasado inadvertidas $\mathrm{y}$, también, se han podido introducir explícitamente las relaciones necesarias y excluir aquellas que eran redundantes. Por otra parte, el propio método axiomático permite determinar cuáles son los axiomas que se pueden eliminar sucesivamente para llegar a teorías cada vez más generales; o, por lo contrario, cuáles axiomas han de agregarse sucesivamente para obtener teoremas cada vez más numerosos y precisos.

\section{Las condiciones que debe cumplir un sistema de axiomas}

El método axiomático ha encontrado su campo de investigación más fecundo en la matemática. En su formalización abstracta, la matemática es tenida como una disciplina que deriva las conclusiones implicadas lógicamente en cualquier grupo de axiomas dado, sin preocuparse por decidir acerca de la validez de su contenido. Lo cual significa que, respecto a los axiomas, lo único importante es que en ellos se apoyen las inferencias que conducen a los teoremas. Por lo tanto, no solamente se consideran vacíos los conceptos que figuran en las proposiciones primitivas, sino que los axiomas mismos resultan carentes de contenido, puesto que solamente representan la estructura de la relación que formulan. En tales condiciones, los axiomas no son verda- 
deros ni falsos. Además, para que un axioma llene su función, no se requiere que cumpla singularmente más condición que la de no ser una proposición auto-contradictoria. En cambio, en su conjunto, el grupo de axiomas tiene que satisfacer tres condiciones ineludibles, que son la consistencia, la integridad y la independencia del sistema que constituyen. De esta manera, la formalización axiomática implica la certeza de que la matemática se funda en la posibilidad de demostrar que el grupo de axiomas establecidos forma un sistema tal que no conduzca a contradicciones $y$, a la vez, que sea completo y no admita ninguna secuencia lógica que lleve de un axioma a otro. En otras palabras, lo que se exige de un sistema de axiomas es, al contrario de lo expresado por el conocido proverbio, que en el sistema estén todos los axiomas que son $y$, a la vez, que lo sean efectivamente todos los que están así considerados. Sólo que, como ya lo advertía claramente Weyl, esto resulta mucho más fácil decirlo que hacerlo. 8

En rigor, las condiciones exigidas a los sistemas de axiomas constituyen un campo de investigación, cuyo programa fue delineado con precisión por Hilbert. Así, dice que su formalización de los fundamentos de la geometría "es un ensayo para construir la geometría sobre un sistema completo de axiomas; lo más sencillo posible".9 Además, considera que sus "cinco grupos de axiomas. . . no están en contradicción unos con otros, esto es, que no es posible, mediante procedimientos lógicos, deducir de ellos hechos que contradigan a los axiomas establecidos". ${ }^{10}$ Y que, "después de haber reconocido la nocontradicción de los axiomas, interesa investigar si todos ellos son independientes entre sí... (esto es) que no es posible derivar, mediante procedimientos lógicos, ninguna parte esencial de un determinado grupo de axiomas, de los grupos de axiomas precedentes".11 La exigencia de que el sistema sea completo, significa que se deben formular expresamente todos los axiomas, de tal manera que nunca haga falta introducir algún nuevo axioma para la construcción de teoremas. La consistencia del sistema de axiomas impone la condición de que no sea posible establecer por medio de inferencias válidas, alguna proposición que se encuentre en contradicción con otra proposición ya demostrada $y$, por supuesto, menos aún con alguno de los axiomas mismos. La otra exigencia es la de que los axiomas sean lógicamente independientes, esto es, que no sea posible obtener ninguno de ellos como conclusión de una serie de inferencias, partiendo de alguno $o$ algunos de los otros axiomas.

Como puede advertirse, en un sistema consistente, independiente y completo, queda excluida formalmente la posibilidad de resolver un problema

8 Herman Weyl, Philosophy of Mathematics and Natural Science, Princeton, Princeton University Press, 1949, pág. 19.

9 Hilbert, op. cit., pág. 1.

10 Hilbert, op. cit., pág. 38 .

11 Hilbert, op. cit., págs. $4^{1-42}$. 
de dos maneras opuestas. Y también, en tal caso, ante cualquier problema que se plantee, siempre resulta posible llegar a una decisión determinada. En cambio, mientras no se pruebe la independencia, la integridad y la consistencia de un sistema de axiomas dado, seguirá existiendo la posibilidad de llegar a descubrir que un axioma no es tal, o encontrar algún teorema que contradiga a otro, o bien, que haga imprescindible para su demostración el agregar un nuevo axioma. Por lo tanto, la prueba del cumplimiento de esas tres condiciones viene a ser el problema medular de la axiomática. Al propio tiempo, es pertinente señalar que dicho problema atañe igualmente a la 16 . gica, ya que ésta también ha sido axiomatizada y, a la vez, los preceptos canónicos y reglas de inferencia de la lógica formal son parte integrante de todo sistema de axiomas. En consecuencia, la propia lógica tiene que cumplir la exigencia formal de ser un sistema consistente, independiente y completo.

\section{El problema de la consistencia}

Un sistema de axiomas es consistente cuando no contiene axiomas que se contradigan mutuamente, ni tampoco se pueden deducir de él proposiciones contradictorias $y$, por ende, en el caso de que se propongan dos teoremas contradictorios, siempre resulte que por lo menos uno de ellos no se pueda demostrar. Así, cuando un sistema de axiomas es consistente, la aplicación del precepto canónico de no-contradicción lleva a establecer que, entre dos proposiciones contradictorias, una de ellas debe ser falsa. En suma, un sistema es consistente cuando, y sólo cuando, nó implica proposiciones contradictorias. En cambio, en el caso de que un sistema de axiomas admita la demostración de dos proposiciones contradictorias, entonces dicho sistema es inconsistente. Además, si tanto una proposición como su contradictoria son deducibles de un sistema de axiomas, resulta que de dicho sistema también es deducible cualquier otra proposición. Por lo tanto, cuando un sistema de axiomas es inconsistente, entonces todas las proposiciones que se puedan postular son demostrables y, por ello, resultan ser teoremas de la disciplina fundada en ese sistema de axiomas. Por consiguiente tenemos, recíprocamente, que un sistema de axiomas es consistente cuando no toda proposición es un teorema, o sea, cuando existe por lo menos una proposición que no sea deducible de los axiomas. De aquí que una prueba de la consistencia de un sistema de axiomas sea la demostración de que existe al menos una proposición que no se puede derivar de los axiomas de ese sistema.

En realidad, cuantas veces se emplea el método axiomático, se plantea el problema de encontrar la prueba de su consistencia; ya que la conjunción de proposiciones inconsistentes implica que de ese mismo sistema es posible obtener cualquier proposición y, por consiguiente, no sólo hace que se derrumbe el sistema, sino que lo disuelve por entero. En el caso de las teorías 
axiomatizadas de la física, la prueba de su consistencia se ha conseguido mediante su interpretación o representación en un modelo objetivo concreto, en cuyo caso es posible investigar si los teoremas son verdaderos en relación con los procesos reales a los cuales se refiere. Pero, en un sentido estrictamente formal, se trata de una prueba relativa, ya que el problema no queda resuelto por el hecho de que los teoremas ya deducidos no se contradigan entre sí, puesto que siempre queda abierta la posibilidad de que un nuevo teorema viniera a poner al descubierto la inconsistencia del sistema. Por eso se ha buscado como medio de probar la consistencia de los sistemas axiomáticos, el reducirlos a los axiomas de una teoría matemática. Ahora bien, para los propósitos de la geometría, es suficiente con el hecho de que las condiciones establecidas por los axiomas de la geometría euclidiana sean consistentes. $\mathrm{Y}$, en efecto, el mismo procedimiento de representación ha mostrado que no puede haber inconsistencia en los axiomas de la geometría elíptica o hiperbólica, a menos que también la haya en los axiomas de la geometría euclidiana que constituyen su modelo. De esta manera, se recurre a la consistencia del sistema euclidiano, como prueba de la consistencia de los sistemas que no son consistentes con la geometría euclidiana. También se puede recurrir a la representación en el sistema de axiomas de la aritmética. Pero, en cualquier caso, siempre vuelve a surgir el problema de probar la consistencia de otro sistema de axiomas. Y, lo que es más, no sólo se requiere probar la consistencia de un conjunto de axiomas, sino del sistema formado por ese conjunto y los preceptos y reglas de la lógica formal. Es decir, debido a que cualquier sistema de axiömas es una transcripción de los teoremas de la lógica, resulta que el problema de probar que dicho sistema es consistente, es equivalente al problema de encontrar la prueba de la consistencia del sistema de axiomas de la lógica formal.

Para precisar el problema, se ha establecido una distinción entre la consistencia formal y la consistencia intrínseca. ${ }^{12}$ De acuerdo con ella, un sistema de axiomas para el cual existe una interpretación, es un sistema interpretable o formalmente consistente. $\mathrm{Y}$, recíprocamente, un sistema de axiomas que no admite ninguna interpretación, es un sistema no-interpretable o formalmente inconsistente. Por otra parte, un sistema de axiomas es intrínsicamente consistente si, después de haber obtenido de él todas sus consecuencias lógicas, jamás se llega a una contradicción, en el sentido de que nunca se pueda deducir simultáneamente la verdad y la falsedad de la misma proposición. Por consiguiente, si un sistema de axiomas es interpretable, entonces es intrínsecamente consistente; pero la inversa no se cumple siempre. En todo caso, para poder utilizar un sistema de axiomas es ineludible que sea intrínsecamente consistente. Porque, si el sistema es intrínsecamente inconsistente,

12 P. S. Novikov, Elements of Mathematical Logic, Londres, Addison-Wesley, 1964. página 100. 
entonces se puede demostrar con base en él una proposición conjuntamente con su negación contradictoria, ya que se pierde así la diferencia entre la verdad y la falsedad; de lo cual resulta que se puede probar la verdad de cualquier proposición. Entonces, el problema de la prueba de la consistencia intrínseca de un sistema de axiomas, es equivalente al problema de obtener la prueba de que dicho sistema es interpretable, o sea, formalmente consistente.

\section{El problemia de la integridad}

Un sistema de axiomas es completo o íntegro cuando contiene todas las proposiciones necesarias y suficientes para deducir de ellas la totalidad de los teoremas expresables dentro de la disciplina fundada en dicho sistema. Así, cuando Euclides formalizó la geometría, indudablemente seleccionó los axiomas de tal manera que fuera posible deducir de ellos todos los teoremas conocidos y, también, aquellos otros que se pudieran establecer después. Además, antes de que se profundizara en el problema, se consideraba que tanto el sistema axiomático de la geometría como el de la aritmética, eran completos o que, en caso de no serlo, por lo menos eran completables agregándoles un número finito y reducido de axiomas. En rigor, la integridad significa que toda proposición perteneciente a una disciplina se ha de poder derivar, lógicamente, de su respectivo sistema de axiomas. Por lo tanto, cuando un sistema de axiomas es completo, entonces, aplicando el precepto canónico de la exclusión de tercero, resulta que entre dos proposiciones contradictorias siempre será posible demostrar que una de ellas es verdadera. En suma, un grupo de axiomas constituye un sistema cuando no falta ninguna de las proposiciones que son axiomas. Sin embargo, en la teoría de los números, por ejemplo, se tienen varias conjeturas famosas que no han podido ser demostradas ni tampoco refutadas. Una de ellas es la hipótesis de Fermat ${ }^{13}$ de que no existen cuaternas de números naturales, $x, y, z, n$, mayores que cero y tales que con ellos se cumpla la relación:

$$
x^{n+2}+y^{n}+2=z^{n}+2 .
$$

Otra es Ia conjetura de Goldbach ${ }^{14}$ acerca de que todo número entero mayor que dos, si es par, es igual a la suma de dos números primos $\mathrm{y}$, cuando es impar, entonces es un número primo o es igual a la suma de tres números primos. $\mathrm{Y}$ así hay otras más, tanto en la teoría de los números como en

13 Précis des Oeuvres Mathématiques de Pierre de Fermat et de l'Arithmétique de Diophante, París, E. Brassinne, 1853, págs. 53-54.

14 P. H. Fuss, editor, Correspondance mathématique et physique de quelques cellebres géometres du XVIIIième siècle, Carta IX de Leonhard Euler a Christian Goldbach, de 10 de agosto de ${ }^{1732}$, pág. 37 . 
otras disciplinas matemáticas. Pues bien, tal vez esas proposiciones son verdaderas, pero también es posible que sean falsas. El hecho es que ni una cosa ni la otra puede ser probada en ninguno de los sistemas de axiomas establecidos hasta ahora. En consecuencia, dichas proposiciones son indecidibles, ya que no se pueden demostrar ni refutar, ni tampoco se puede probar que sean indemostrables o irrefutables.

Con vistas a precisar el signifcado que tiene la integridad de un sistema de axiomas, se distingue entre su consideración en sentido amplio y en sentido estrecho. ${ }^{15}$ Así, en sentido amplio, un sistema de axiomas es completo cuando toda proposición tautológica perteneciente a la disciplina respectiva es deducible de dicho sistema. En otras palabras, un sistema de axiomas es completo en sentido amplio, cuando contiene los axiomas y reglas suficientes para deducir una proposición arbitraria cualquiera que, en la interpretación formal del propio sistema, sea una tautologia. En caso contrario, el sistema de axiomas es incompleto. Por otra parte, un sistema de axiomas es completo en sentido estrecho cuando, al agregarle en calidad de axioma cualquier proposición que no sea deducible del sistema original, se obtiene como resultado una contradicción. A la vez, un sistema de axiomas es formalmente completo cuando todas sus interpretaciones son isomórficas, o sea, cuando entre los elementos de todas las parejas de interpretaciones del sistema existen relaciones biunivocas. En fin, un sistema de axiomas es intrínsecamente completo cuando todas las proposiciones que son verdaderas, para cualquiera de sus interpretaciones arbitrarias, son proposiciones deducibles del mismo sistema. Por consiguiente, si un sistema de axiomas es formalmente completo, entonces también es intrínsecamente completo; pero la reciproca no siempre es cierta. Ahora bien, lo que quedaba planteado era el problema de probar que un sistema de axiomas establecido fuera completo o, por lo menos, que resultara completable con un número reducido de nuevos axiomas.

\section{El problema de la independencia}

En un sistema de axiomas no deben figurar proposiciones innecesarias que puedan deducirse de los restantes axiomas, ya que entonces deben estar incluidas entre los teoremas. Cuando se tiene un sistema de axiomas en el cual todos ellos son efectivamente proposiciones primitivas no deducibles recíprocamente, se tiene un sistema independiente o un sistema de axiomas mutuamente independientes. Por lo tanto, de la misma manera en que un sistema es completo cuando no le falta ningún axioma, así también, dicho sistema es independiente cuando no le sobra ningún axioma. Un axioma es independiente dentro de un sistema, cuando no se puede deducir de los otros axiomas mediante las reglas de inferencia admitidas. De no ser así, dicho axio-

15 Novikov, op. cit., págs. $77-79,151-154$. 
ma es dependiente. Por su parte, un sistema de axiomas en el cual ninguno de ellos es deducible de los otros, es un sistema de axiomas independiente. En caso contrario, el sistema de axiomas es dependiente. En todo caso, para probar la independencia de un axioma respecto a los demás del sistema, se requiere demostrar que ni tal axioma, ni su opuesto contradictorio, ni todas las proposiciones derivadas de ellos, se encuentran en contradicción con los otros axiomas del sistema. Los axiomas dependientes pueden ser eliminados del sistema, sin que se altere el conjunto de teoremas de la disciplina de que se trate; ya que, en último término, si un axioma no es independiente, entonces realmente no es un axioma sino un teorema. Por otro lado, cuando la consistencia interna de un sistema de axiomas sólo se puede probar recurriendo a otro sistema de axiomas, entonces tenemos que, en rigor, el primer sistema es dependiente del segundo. Aparentemente, un sistema de axiomas no deja de ser válido cuando se descubre que uno de sus axiomas se puede deducir de los otros; y, en tal caso, lo que debe hacerse obviamente es excluirlo del sistema de axiomas y pasarlo a su calidad de teorema. Sin embargo, simplemente para comprender la estructura de cualquier sistema de axiomas, es indispensable probar su independencia. $\mathrm{Y}$, lo que es más importante aún, la falta de independencia de los axiomas de un sistema significa, estrictamente, que todas las proposiciones de la disciplina en cuestión son teoremas. En tal caso, se disuelve lógicamente el sistema de axiomas establecido y, al propio tiempo, resulta posible seleccionar arbitrariamente diversos grupos de teoremas, para habilitarlos como axiomas, aunque a sabiendas de que ninguno de esos grupos constituye realmente un sistema de axiomas.

Con objeto de precisar el significado que tiene la independencia de los axiomas, se puede distinguir entre la independencia formal y la independencia intrínseca. ${ }^{16}$ De esa manera, un axioma es formalmente independiente de los otros axiomas de un sistema, cuando existe un conjunto de teoremas que satisface el sistema de axiomas formado por esos otros axiomas sin el primero, pero que no satisface el sistema de axiomas original, o sea, el sistema en el cual figura además el axioma en cuestión. En cambio, un axioma es intrínsecamente independiente de los otros axiomas de un sistema, cuando no se puede deducir de esos otros axiomas. Entonces, si un sistema es formalmente independiente, también es intrínsecamente independiente; pero la recíproca no siempre es válida. Por otra parte, un axioma es dependiente de los otros axiomas de un sistema, cuando una interpretación arbitraria del sistema formado exclusivamente por esos otros axiomas, satisface igualmente al sistema original, en el cual está incluido el axioma en cuestión. Desde luego, la inde pendencia intrínseca es necesaria para evitar que en el sistema se consideren axiomas superfluos. $Y$ la independencia formal es indispensable para que el grupo de axiomas constituya efectivamente un sistema. Pues bien, como

16 Novikov, op. cit., págs. 79-87, $101-102$. 
lo veremos después, lo que Cohen acaba de demostrar es justamente que no se puede probar la independencia de ningún sistema de axiomas.

El problema de la independencia de un axioma perteneciente a un sistema, respecto a los otros axiomas, conduce a otro problema de primordial importancia, como es la posibilidad de sustituir dicho axioma por su negación, sin causar una contradicción interna en el sistema en cuestión. Como primer ejemplo de este problema, tenemos el de la independencia del quinto postulado de Euclides en el sistema de axiomas de la geometría. Como ya to hemos dicho, ese descubrimiento condujo a la formulación de las geometrias no-euclidianas, cuyos axiomas son los mismos de la geometría euclidiana, con excepción del relativo a las paralelas, que se sustituye por la negación contradictoria del postulado de Euclides. Por lo tanto, de esa manera se puso en claro que si un sistema de axiomas es consistente, y sus axiomas son independientes, entonces el sistema sigue siendo consistente cuando se sustituye uno de los axiomas por su contradictorio. Más adelante, Hilbert, en su empeño por realizar el programa que se había trazado, intentó demostrar la independencia de los 20 axiomas de su sistema de geometría "por medio de ciertas geometrías enteramente peculiares, construidas ad hoc".17 Para ello, intentó demostrar que cada axioma era independiente de los otros, valiéndose de la construcción de un modelo en el cual se satisfacen todos los axiomas del sistema, con excepción del axioma en cuestión. Así, empezó por inferir la independencia del axioma de las paralelas, por medio de un modelo conocido de la geometría no-euclidiana; luego siguió con el axioma sobre el transporte de segmentos de recta, introduciendo una nueva definición de la congruencia, y así sucesivamente. ${ }^{18}$ Pronto haremos ver que tales pruebas no son suficientes, de acuerdo con la demostración de Cohen. Mientras tanto, precisaremos el significado que tiene la independencia de un axioma, en relación con el problema de la posibilidad de sustituirlo por su opuesto. Así tenemos que un axioma perteneciente a un sistema de axiomas es dialécticamente independiente cuando, y sólo cuando, además de ser satisfactorio el sistema de axiomas original, también resulta satisfactorio el sistema de axiomas formado por los otros axiomas más el opuesto al axioma en cuestión.

\section{Los trabajos de Goedel}

En $19^{\circ} 4$, Hilbert señaló que la lógica simbólica podía ser tratada como una rama de la teoría de los números. ${ }^{19}$ Y Goedel fue el primero en elaborar en detalle esa correspondencia, utilizándola para investigar las propiedades

17 H. Weyl, "David Hilbert, 1862-1943"; Obituary Notices of Fellows of the Royal Society, 4, 1942-1944, págs. 547-553.

18 D. Hilbert, op. cit., págs. 41-55.

19 D. Hilbert, "Ueber die Grundlagen der Logik und Arithmetik", Verhandlungen des Dritten Internationalen Mathematiker-Kongresses in Heidelberg, 1904, págs. 174-185. 
de los sistemas formalizados de axiomas. En efecto, en 1930, Goedel publicó la primera prueba de la independencia del sistema de axiomas constituido por las reglas de la lógica deductiva, demostrando que no es completo en sentido amplio, pero si lo es en el sentido estrecho y, por lo tanto, resulta suficiente para la demostración de los esquemas formales de la lógica. ${ }^{20}$ Al año siguiente, Goedel publicó su trabajo fundamental y ahora famoso, en el cual ofreció las pruebas de que ningún sistema de axiomas puede ser completo, ni tampoco puede ser consistente y, por ende, puso al descubierto dos de las tres limitaciones insuperables que son inherentes al método axiomático. ${ }^{21}$ Con ese trabajo, terminó la etapa del formalismo axiomático dominada por el programa y los resultados de Hilbert, que fue intensamente creadora y bastante fructuosa, y durante la cual se desarrollaron muchas concepciones básicas y técnicas nuevas, pero que resultó no ser suficientemente crítica respecto a sus fundamentos y se caracterizó por la ignorancia de sus limitaciones. Al propio tiempo, las demostraciones de Goedel desencadenaron esa profunda crisis en la axiomática, que ahora ha venido a culminar con la prueba de Cohen. En la imposibilidad de realizar el programa trazado por Hilbert, la axiomática entró en una fase más reflexiva y, en particular, agudamente crítica en relación con el formalismo.

La demostración de Goedel se encuentra expuesta en detalle en varios trabajos conocidos, ${ }^{22}$ por lo cual nos limitaremos a dar aquí una idea de ella. En primer lugar, Goedel formula un criterio de consistencia intrínseca formalmente más riguroso que el utilizado anteriormente, al que llama consistencia-omega. ${ }^{23}$ Luego adopta un sistema de representación, en el cual a cada símbolo usado en las fórmulas matemáticas le corresponde biunívocamente un número natural. Así, cualquier fórmula, que es simplemente una colección finita de símbolos, queda representada por una colección de núme-

20 Kurt Goedel, "Die Vollstaendigkeit der Axiome des Logischen Funktionkalkuels", Monatshefte fuer Mathematik und Physik, 37, 1930, págs. 349-960.

21 K. Goedel, "Ueber formal unentscheidbare Saetze der Principia Mathematica und verwandter Systeme", Monatshefte fuer Mathematik und Physik, 38, 1931, págs. 173-198.

22 Además del artículo original de Goedel, cuya referencia damos en la nota 21 , se pue. den consultar los siguientes trabajos: K. Goedel, On Undecidable Propositions of Formal Mathematical Systems, notas mimeográficas de sus conferencias en el Instituto de Estudios Avanzados, tomadas por S. C. Kleene y J. B. Rosser, Princeton, 1934; J. B. Rosser, "An informal exposition of proofs of Goedel's theorems and Church's theorem", Journal of Symbolic Logic, 4, 1939, págs. 53-60; A. Mostowski, Sentences Undecidable in Formalized Arithmetic. An Exposition of the Theory of. Kurt Goedel, Amsterdam, North-Holland, 1952; N. R. Hanson, "The Goedel theorem. An informal exposition", Notre Dame Journal of Formal Logic, 2, 1961, págs. 94-110; E. Nagel y J. R. Newman, Goedel's Proof, en The Mathematical Way of Thinking, ed. por J. R. Newman, Nueva York, Simon and Schuster, 1956, págs. 16681695 (La prueba de Goedel, Cuadernos del Centro de Estudios Filosóficos, México, U.N.A.M., Núm. 6, traducción de Ramón Xirau, 1959).

23 El cual permitió a Goedel demostrar que si una cierta fórmula es demostrable, entonces su negación contradictoria es demostrable; y si esa negación contradictoria es demostrable, entonces el sistema formalizado al que pertenece es inconsistente-omega. 
ros naturales y, como la correspondencia es biunívoca, en cualquier momento se puede convertir de nuevo la colección de números en la fórmula original. Además, las reglas para la construcción de nuevas fórmulas y la conjugación de éstas, se expresan en función de las operaciones aritméticas elementales ejecutadas con los números representativos. De esa manera, todas las proposiciones son transformadas uniformemente en fórmulas aritméticas. Entonces, Goedel construye una fórmula aritmética tal que solamente es demostrable cuando su negación también es demostrable. $O$ sea, que se trata de una fórmula que, en rigor, no es demostrable ni refutable. En consecuencia, dicha fórmula es formalmente indecidible $y$, por lo tanto, el sistema de axiomas de la aritmética es incompleto. Más aún, el sistema de la aritmética es incompletable; de tal manera que, aún cuando sea posible seguir agregando nuevos axiomas, subsistirá la misma situación, ya que siempre se podrá construir otra fórmula que resulte indecidible. Al propio tiempo, Goedel demostró que, incluso suponiendo que un sistema de aritmética formalizada sea consistente, no es posible dar una prueba de su consistencia que pueda ser formalizada dentro del mismo sistema de la aritmética. Entonces, dicha consistencia sólo puede ser demostrada recurriendo a una teoría matemática superior, como lo es la teoría de los números transfinitos, por ejemplo; cuya consistencia deberá ser probada, a su vez, refiriéndola a otra teoría matemática más compleja, y así sucesivamente. Por lo tanto, la prueba de la consistencia interna de la matemática se complica cada vez más $y$, finalmente, sigue siendo tan dudosa como al principio, ya que el procedimiento de prueba es interminable.

Después de probar la existencia de proposiciones indecidibles, Goedel estableció dos condiciones simples para ampliar el dominio de aplicación de sus descubrimientos, las cuales expresó de la síguiente manera: $I$. La clase de los axiomas y las reglas de inferencia deben ser definibles recursivamente. 2. Cada relación recursiva debe ser definible dentro del sistema. Entonces, demostró que cualquier sistema formal que cumpla esas dos condiciones y sea consistente, en el sentido omega, contiene proposiciones indecidibles. Entre esos sistemas se encuentran, desde luego, la axiomatización formulada por Zermelo-Fraenkel para la teoría de los conjuntos, y el sistema de la aritmética constituido por los axiomas de Peano, las definiciones recursivas y las reglas de la deducción formal. Además, debido a que la demostración de la consistencia interna de cualquier teoría formalizada de una disciplina científica, se establece mediante su reducción a los axiomas de la aritmética, entonces resulta que las pruebas de Goedel se refieren a cualquier teoría formalizada y, por ende, a todo sistema de axiomas que se pueda formular. En consecuencia, todo sistema de axiomas es necesariamente incompleto e incompletable. Y, al propio tiempo, en el caso de que se suponga que tiene consistencia interna, ésta es indemostrable dentro del sistema mismo. Más 
todavia, como cualquier teoría formalizada incluye ineludiblemente a las reglas de la inferencia deductiva, como partes integrantes de su sistema, queda en claro que las pruebas de Goedel afectan igualmente a la lógica formal.

\section{La prueba de Cohen}

El cambio de orientación en las investigaciones, como consecuencia de los resultados obtenidos por Goedel, llevó a examinar el problema de la consistencia relativa de los sistemas de axiomas. Esto es, en lugar de una prueba absoluta de la consistencia de un sistema formal, se buscó la manera de probar que, si un sistema de axiomas se puede tener como consistente, entonces también es consistente otro sistema de axiomas que sea una extensión del primero. De nuevo, fue Goedel quien logró encontrar esa demostración, ${ }^{24}$ probando rigurosamente que si es consistente la formalización de la teoría de los conjuntos establecida por Zermelo-Fraenkel, ${ }^{25}$ pero sin incluir el axioma de selección, entonces sigue siendo consistente la teoría formada cuando se agregan el axioma de selección ${ }^{26}$ y la hipótesis del continuo de Cantor generalizada.27 Pues bien, esta demostración constituye la base utilizada por Cohen para dar el siguiente paso, que ha sido definitivo. En efecto, la prueba de Cohen consiste justamente en demostrar que: "La hipótesis del continuo no puede ser derivada de los otros axiomas de la teoria de los conjuntos, incluyendo entre ellos el axioma de selección. Y, como Goedel ha demostrado ya que la hipótesis del continuo es consistente con dichos axiomas, entonces queda establecida asi la independencia de la hipotesis del continuo." 28 Cohen trabaja primero con el sistema de axiomas que Goedel denomina $(Z-F)$ y el cual consta de los axiomas usuales de la teoría de los conjuntos de Zermelo-

$24 \mathrm{~K}$. Goedel, The Consistency of the Axiom of Choice and of the Generalized Continuum Hypothesis with the Axioms of Set Theory, Princeton, Princeton University Press, 1940.

25 Los axiomas usuales de esta teoria son los de: determinación (Bestimmtheit); conjuntos elementales (Elementarmengen); separación (Aussonderung); potenciación (Potenzmenge); unión (Vereinigung); selección (Auswahl); infinitud (Unendlichen); y sustitución (Ersetzung).

26 El axioma de unión establece que a cada conjunto le corresponde un conjunto-unión que contiene como elementos a todos los elementos de los elementos de dicho conjunto y sólo a ellos. El axioma de selección establece que si todos los elementos de un conjunto son conjuntos no vacios y que no tienen ningún elemento en cornún, entonces su conjuntounión correspondiente contiene, por lo menos, un subconjunto que tiene un elemento, y sólo un elemento, en común con cada elemento del conjunto original.

27 La conjetura de Cantor o hipótesis del continuo establece que el número transfinito que expresa el conjunto de los números reales, simbolizado por ale $f_{1}$, es igual al número 2 elevado a alef $_{0}$, que es el transfinito que representa el conjunto de los números naturales, o sea que: alef ${ }_{1}=2^{\text {alef }}$. Mientras que la hipótesis del continuo generalizada establece esa misma relación entre cualquier transfinito y el transfinito de orden inmediato superior, esto es: alef $+1=2_{r}{ }^{a l \theta f}$.

$28 \mathrm{~J} . \mathrm{P}$. Cohen, op. cit. 
Fraenkel, pero sustituyendo el axioma de selección por el axioma de regularidad. ${ }^{29}$ Entonces demuestra el siguiente Teorema:

Existen modelos para el sistema $(Z-F)$, en los cuales sucede que:

1) existe un conjunto tal que no es construible, ${ }^{30}$ no obstante que se cumplen tanto el axioma de selección como la hipótesis del continuo generalizada;

2) el continuo no es un conjunto bien ordenado;

3) se cumple-el axioma de selección, pero no se cumple la hipótesis del continuo de Cantor;

4) no se cumple el axioma de selección para ciertas parejas enumerables de elementos.

Por consiguiente, "como el axioma de constructibilidad implica la hipótesis del continuo generalizada, y ésta implica a su vez el axioma de selección, resulta que dicho Teorema establece manifiestamente la cuestión de la validez relativa de dichos axiomas". 31

Por otra parte, Cohen trabaja también con el sistema $(Z-F)^{\prime}$, que denota el sistema de axiomas que se obtiene de agregar al sistema $(Z-F)$ otro axioma, que es el siguiente:

Existe un conjunto $m$ que es un modelo para el sistema $(Z-F)$. En tal caso, según lo demostró Goedel, ${ }^{32}$ del sistema $(Z-F)^{\prime}$ se puede deducir la existencia de un conjunto $n$ que es un modelo para el sistema (Z-F). Pues bien, Cohen demuestra, a su vez, que: 'El sistema $(Z-F)^{\prime}$ ' además de la existencia del conjunto $n$, que es un modelo para el sistema $(Z-F)$, también implica el cumplimiento del axioma de selección y de la negación de la hipótesis del continuo. $\mathrm{Y}$, por lo tanto, ... se puede considerar firmemente establecida la indemostrabilidad de la hipótesis del continuo." 33 Al propio tiempo, Cohen ha logrado poner al descubierto que entre el transfinito alefo, que representa el número de los números naturales, y el transfinito ale $f_{1}$, que representa el número de los números reales, existe por lo menos otro número transfinito; lo cual quiere decir que ale $f_{1}$ no es el transfinito inmediato de orden superior a ale $_{0}$. De esta manera, Cohen ha dejado establecida rigurosamente la prueba de la independencia de la hipótesis del continuo, como

29 El axioma de regularidad (Fundierung), formulado por John von Neumann, establece que todo conjunto no vacio $s$, contiene un elemento $t$ tal que $s$ y $t$ no tienen ningún elemento en común.

30 Un conjunto es construible cuando, además de existir, se puede construir deductivamente.

31 J. P. Cohen, op. cit.

32 K. Goedel, The Consistency of the Continuum Hypothesis.,

33 J. P. Cohen, op. cit. 
parte integrante del sistema de axiomas de la teoría de los conjuntos. Entonces, como ya lo apuntábamos al principio, la prueba de Cohen muestra que se puede sustituir la hipótesis del continuo por su negación contradictoria, sin causar una inconsistencia en el sistema de axiomas de la teoría de los conjuntos, porque se trata de un axioma que es dialécticamente independiente. Por lo tanto, se han reunido los elementos fundamentales necesarios para formular una nueva teoría de los conjuntos y, en realidad, nuevas teorías de las otras ramas de la matemática, a las cuales podemos denominar provisionalmente como no-cantorianas, en un sentido enteramente análogo al que tiene la geometría no-euclidiana en relación con la euclidiana. A la vez, debido a que el fundamento mismo de la prueba es la consistencia relativa del sistema de axiomas, resulta que Cohen ha venido a probar igualmente que es imposible demostrar la independencia de los axiomas integrantes de cualquier sistema formalizado. Por consiguiente, la prueba de Cohen significa que tampoco es demostrable estrictamente el cumplimiento de la tercera y última condición, que se planteaba como exigencia ineludible para que un sistema de axiomas sea enteramente riguroso.

La demostración de que no es posible probar la consistencia, ni la integridad, ni tampoco la independencia de un sistema de axiomas, pone claramente de manifiesto que los axiomas son insuficientes para caracterizar las teorías de la matemática $y$, en general, de cualquier otra disciplina científica. Como lo dice el propio-Goedel, "existe, en algún sentido, una realidad objetiva que los matemáticos tratan de expresar en los axiomas de la teoría de los conjuntos, pero los axiomas formulados hasta ahora sólo representan esa realidad de un modo incompleto". 34 Antes del descubrimiento de Cohen, todavía era posible considerar que esta situación se podría superar mediante la introducción de otros axiomas, tal vez muy diferentes de los actuales, que sirvieran para representar la realidad objetiva de una manera más aproximada y menos incompleta. Pero, ahora sabemos bien que cualquier interpretación axiomática que se intente, será siempre una representación insuficiente y limitada de la realidad. Más todavía, ha quedado refutada la afirmación que se hacía con frecuencia, acerca de que la formalización axiomática de las disciplinas cientificas era lógicamente inevitable, apodícticamente verdadera y completamente independiente de la experiencia. Así, se ha consumado definitivamente y sin remedio el derrumbe de la axiomatización, en el sentido apriorístico y absolutizante con que era considerada. Por lo tanto, debemos concluir que, respecto al problema de la axiomatización ha sucedido lo mismo que antes ocurrió con otros problemas matemáticos, como el de encontrar la cuadratura del círculo o la trisección de un ángulo empleando exclusivamente regla y compás, el de resolver la ecuación de quin-

34 K. Goedel, "What is Cantor's Continuum Problem?", American Mathematical Monthly, 54, 1947, págs. 515-525. 
to grado por medio de radicales, el de la construcción de números trascendentes por medios algebraicos, y el de la demostrabilidad del axioma de las paralelas. Esto es, que la axiomatización es un problema imposible de resolver utilizando únicamente la lógica formal, al igual que aquellos otros problemas tampoco pudieron ser resueltos por los medios con que se intentaban. Sólo que, como lo señala acertadamente Hilbert, en la ciencia "continua desempeñando un papel preponderante la imposibilidad de ciertas soluciones o problemas, y el esfuerzo realizado para contestar a una cuestión de tal índole, ha sido con frecuencia la causa del descubrimiento de un nuevo y fructífero campo de investigación".35

\section{Consecuencias y perspectivas}

La primera consecuencia que se desprende inmediatamente de la prueba de Cohen es que jamás se logrará construir deductivamente una disciplina cientifica completa, consistente e independiente. Porque se ha puesto de manifiesto que las ciencias, y en particular la matemática, tienen la propiedad intrínseca de no poder ser formalizadas por entero. Por lo tanto, el propio conocimiento científico se niega a quedar aprisionado en ese mundo de abstracción total, constituido exclusivamente por sistemas de axiomas y reglas interdependientes, en donde cualquier teoría que se estableciera arbitrariamente, resultaba ser tan buena y aceptable como otra cualquiera. Al propio tiempo, de la misma manera en que las pruebas de Goedel señalan destacadamente el incumplimiento de los preceptos de no-contradicción y de exclusión de tercero, así también la demostración de Cohen significa el derrumbe de la lógica formal, en cuanto a su carácter absoluto, precisamente dentro del último reducto en donde era cultivada en ese sentido. Por consiguiente, tenemos que las concepciones y los más refinados medios de investigación basados en la lógica formal, no pudieron satisfacer las severas exigencias que plantea la teoría de los conjuntos y, con ella, las otras disciplinas matemáticaś. Ciertamente, la lógica de la matemática supera a la lógica formal y, especialmente en los momentos críticos de su desarrollo, exhibe conspicuamente su carácter dialéctico. En rigor, las pruebas de Goedel y Cohen son un resultado de la dialéctica de la matemática y abren nuevos cauces para el desenvolvimiento de la lógica dialéctica.

Por otra parte, de la prueba de Cohen se desprende igualmente que los procedimientos matemáticos demostrativos no coinciden, ni menos se agotan, con el método axiomático. Lo cual quiere decir que los recursos de la razón no pueden ser formalizados por completo y que tampoco es posible fijar ninguna limitación a la capacidad creadora de los matemáticos y los lógicos para inventar, descubrir y desarrollar nuevos procedimientos de demostra-

35 D. Hilbert, Grundlagen der Geometrie, pág. 134. 
ción. A este problema se encuentra ligada la cuestión de que existe un gran número de proposiciones matemáticas verdaderas que, sin embargo, no se pueden deducir formalmente de ningún grupo dado de axiomas, mediante el empleo del grupo cerrado de reglas de la inferencia deductiva. Por la imposibilidad de probar la consistencia, resulta la existencia de esas proposiciones indecidibles, que no se pueden demostrar ni refutar. Del hecho de que ningún grupo de axiomas sea completo, y además no sea completable, proviene la dificultad insuperable que se presenta para la demostración de todas las proposiciones pertenecientes a una teoría. Y, la falta de independencia de los axiomas, significa que no es posible distinguir entre conceptos básicos y conceptos derivados, ni entre relaciones fundamentales y relaciones secundarias; por lo cual, tampoco es posible distinguir realmente un axioma de un teorema. Más aún, la imposibilidad de probar la independencia lleva a la situación de que, en rigor, todas las proposiciones son teoremas y, entonces, se puede escoger con libertad entre una infinidad de grupos de proposiciones, para otorgarles arbitrariamente el rango de axiomas. Esta situación comprende hasta los mismos preceptos canónicos o "principios lógicos supremos", puesto que también constituyen un sistema de axiomas $\mathrm{y}$, por ende, resulta que dichos "principios" tienen que ser considerados, en principio, como inconsistentes, incompletos y dependientes. A la vez, debido a que las reglas formales de inferencia son parte integrante de todos y cada uno de los sistemas axiomáticos establecidos hasta ahora, quedan igualmente incluidas en la falta de consistencia, de integridad y de independencia. Por lo tanto, se impone de manera imperiosa la necesidad de emplear otras reglas de transformación, diferentes y más poderosas que las reglas de la deducción formal. A este respecto, las demostraciones de Goedel y Cohen constituyen el fundamento teórico necesario para la formulación de la teoría matemática de la logica dialéctica, cuyo desarrollo permitirá seguramente hacer más rigurosas y eficaces las operaciones ya conocidas y, a la vez, descubrir nuevas formas dialécticas de razonamiento.

En lo que respecta a la matemática, la prueba de Cohen abre un campo de investigación inmenso y promisorio, a la veż que obliga a cambiar decididamente la orientación seguida dentro de una de las tendencias dominantes de la matemática contemporánea y viene a desencadenar una revolución en todas sus ramas. Desde luego, con la relativización del formalismo se destaca la imposibilidad de separar la forma del contenido, ya que éste gobierna el progreso de aquélla y decide su importancia y las modalidades que adquiere. Al propio tiempo, se pone en evidencia cómo la acción de descubrir sobre el papel, con lápiz o pluma y por medio del razonamiento, las relaciones matemáticas que constituyen una representación aproximada de las relaciones objetivas, solamente resulta posible cuando el conjunto de axiomas del cual se parte es también una imagen de la realidad objetiva. El método 
axiomático es únicamente una forma de representación que requiere, de manera indispensable, una fundamentación objetiva. En todo caso, la axiomatización ha quedado reducida a sus justos términos, o sea, que es una actividad secundaria y subsecuente al descubrimiento de las relaciones fundamentales estudiadas dentro de cada disciplina, y por medio de la cual se expresan en una forma precisa. La tarea de axiomatización es relativa y sus criterios de rigor se encuentran sujetos a cambios, de acuerdo con el desenvolvimiento del conocimiento científico. Entonces, se puede seguir considerando que la formalización axiomática de una disciplina constituye la formulación más rigurosa que puede hacerse de ella en un momento dado, con tal que se tenga siempre en cuenta que se trata simplemente de una forma de expresión de los conocimientos logrados y que las exigencias lógicas que debe cumplir la conexión de sus axiomas y teoremas, también se encuentran sujetas a evolución. Y, por cierto, lo que en este momento se plantea con una necesidad imperiosa e inaplazable, es la elaboración de los nuevos criterios de rigor que deban sustituir a las frustradas exigencias de la formalización axiomática:

En lo que se refiere a la lógica, se podrá desenvolver la teoría matemática de la dialéctica, cuyo fundamento queda establecido con las implicaciones que tienen los resultados de Goedel y Cohen. Este desarrollo podrá conducir después a la invención y la construcción de nuevas computadoras electrónicas, que serán superiores a las actuales. En efecto, las computadoras funcionan hasta ahora conforme a las reglas fijas de las operaciones axiomáticas formalizadas, mientras que la lógica dialéctica les impartirá una estructura operativa mucho más rica, fina y penetrante. Más aún, la misma asociación entre la lógica dialéctica y las computadoras, permitirá por vez primera un desenvolvimiento amplio de la lógica experimental. Por lo tanto, el derrumbe definitivo del apriorismo, provocado por la revolución científica que se ha suscitado, conduce directamente a la experiencia, tanto en la matemática como en la lógica. En este sentido ya se vienen realizando algunos trabajos de gran importancia. Entre ellos, podemos mencionar las investigaciones de Church sobre las funciones que son computables sistemáticamente, mismas que lo llevaron primero a establecer que toda función efectivamente calculable es recursiva ${ }^{36} \mathrm{y}$, luego, a descubrir que la definibilidad lambda es equivalente a la recursividad. ${ }^{37}$ Por su parte, Turing ha confirmado esa demostración, precisando el concepto de computabilidad e inventando la máquina que lleva su nombre para probar la computabilidad de las funciones. ${ }^{38} \mathrm{Al}$ propio tiempo, se vienen desarrollando algunos len-

36 A. Church, "A set of postulates for the foundation of logic", Annals of Mathematics, 33, 1932, págs. $346-366$; 34,1933 , págs. $839-864$.

37 A. Church, The Calculi of Lambda-Conversion, Princeton, Princeton University Press, $194^{1}$.

38 A. M. Turing, "On computable numbers, with an application to the Entscheidungs- 
guajes para las computadoras electrónicas, que se encuentran basados por completo en la recursividad. ${ }^{39}$ Incidentalmente, tenemos que ya ha sido posible formular un algoritmo que permite decidir si una fórmula es o no es un teorema; ${ }^{40}$ y su expresión en el lenguaje $L i s p$ ha servido para demostrar en una computadora, en 8 minutos, todos los teoremas que figuran en los Principia Mathematica de Whitehead y Russell. De esta manera, se vienen creando los elementos teóricos y simultáneamente los instrumentos matemáticos de aplicación, que son necesarios para el establecimiento y el desarrollo práctico de la teoría matemática de la lógica dialéctica. Por eso es que podemos concluir, insistiendo vigorosamente en la afirmación de que las consecuencias de las pruebas de Goedel y Cohen inauguran una etapa revolucionaria en la matemática, la lógica y la cibernética.

ELI DE GorTari

problem", Proceedings of the London Mathematical Society, 42, 1936, págs. 230-265; 43, 1937, págs. $544-546$.

30 Véase, por ejemplo, John McCarthy et al., Lisp 1.5 Programmer's Manual, Cambridge, Massachusetts Institute of Technology, 1962.

40 Wang Hao, "Toward Mechanical Mathematics", IBM Journal, 4, 1960, págs. 2-22. 